\title{
Compassionate Limit Setting for the High-Risk Non- Adherent Patient Population
}

\author{
ARIEL CLATTY, PhD \\ ERIN HEDGLEN, MA \\ MA_hedglene@duq.edu
}

\begin{abstract}
In a society that overlooks, stigmatizes, and becomes desensitized to the growing substance use disorder population, medical ethics looks to the future to help patients that are high-risk and non-adherent (HRNA) while in an acute care setting. Medical ethics can create an environment that supports providers in managing patients with behavioral, social, and substance use disorders as well as offering a higher quality of care by providing consistent, safe, and effective care for patients.

This initiative provides a safe and therapeutic environment for patients and healthcare teams. This stance of medical ethics outlines the communication management of non-adherent patients and family members to help aid in the continuation of their inpatient medical treatment. All possible avenues will be explored for the best environment to optimally control the situation, and to provide safe and effective medical care for the patient and lessen the risk to staff. Ethics' goal is to facilitate conflict management discussions between medical providers and the patient. This paper then gives the perspectives of medical ethics conflict management strategies pertaining to a case study based upon the HRNA patient population.
\end{abstract}

\section{INTRODUCTION}

Physicians often face patient requests for medically inappropriate treatment. Such requests can trigger physician ${ }^{1}$ and patient distress and disrupt the therapeutic alliance between care providers and patients. Physician denials of these requests can lower patient satisfaction. ${ }^{2}$ Nevertheless, refusal of requests for inappropriate treatment is ethically warranted given the significant harms such treatment imposes on patients. ${ }^{3}$ Inappropriate opioid use imposes especially challenging patient risks and public health burden. ${ }^{4}$ As these requests are becoming more prevalent, how can providers approach these requests consistent with the principals of medical ethics and instituting patient-centered care? In this clinical analysis of a difficult patient encounter, we recommend a multi-disciplinary approach in order to maintain active patient-physician relationship that is driven by medical ethics principles despite limiting contraindicated opioid therapy.

*Address correspondence to: Erin Hedglen. MA., E-mail: MA_hedglene@duq.edu

+To cite this article: Clatty, Ariel and Erin Hedglen. "Compassionate Limit Setting for the High-Risk Non-Adherent Patient Population" The Journal of Healthcare Ethics \& Administration 6, no. 1 (Spring/Summer 2020)19-23, https://doi.org/10.22461/jhea.1.71624

This work is brought to you for free and open access by the Institute of Clinical Bioethics (ICB) at Saint Joseph's University, Philadelphia, PA, U.S.A. It has been accepted for inclusion in The Journal of Healthcare Ethics \& Administration by the editorial board and an authorized administrator of the JHEA. For more information, please contact support@jheaonline.org

\footnotetext{
${ }^{1}$ Fenton JJ. Et al. Impact of patient requests on provider-perceived visit difficulty in primary care. Pg. 214-220.

2 Jerant A, et al. Association of clinician denial of requests and patient satisfaction. Pg. 285-91.

${ }^{3}$ Misak CJ, et al. Medically inappropriate or futile treatment: deliberation and justification. Pg. 90-114

${ }^{4}$ Bonnie RJ, et al. Pain management and the opioid epidemic.
} 


\section{THE JOURNAL OF HEALTHCARE ETHICS \& ADMINISTRATION}

Vol. 5 | No. 1 (Spring/Summer 2020)

\section{HIGH RISK NON-ADHERENT PATIENTS}

The high risk non-adherent (HRNA) patient population is comprised of the following: patient is high risk for patient safety concerns in the hospital, patient is non-adherent to treatment plan inpatient and outpatient, patient may have high remittance rate, and patient may be verbally aggressive, violent, or visitors may be non-adherent to hospital policies. Some of these patients clinically present similarly to patients with a history of substance abuse. Patients have rights to refuse care, and not follow treatment plans, however this can severely affect the physician-patient relationship and the course of care. Options available to patients who wish not to participate or engage in care such as leaving against medical advice (AMA) should be examined on a case by case basis in terms of risks and harm. ${ }^{5}$ The high risk non-adherent patient population described here are likely to undergo multiple readmissions in their lifetime. These readmissions are not solely dependent on the individuals being "difficult" per treatment teams, but also a failure on behalf of the healthcare system for not doing more to meet the needs of this patient population. A category that relates closely to high risk non-adherent patients may be a group of patients with chronic non-cancer pain. These patients make up almost thirty percent of the United States adult population. It may be said that many HRNA patients have chronic non-cancer pain, however not all chronic non-cancer pain patients are HRNA. ${ }^{6}$ Chronic non-cancer pain (CNCP) may be defined as pain that persists for longer than three months and is not caused by a malignancy or associated with pain at the end of life. ${ }^{7}$ Chronic pain, may alter how a patient perceives the healthcare system. CNCP is often diagnosed in patients that may have a co-existing substance use disorder. ${ }^{8}$ Healthcare systems are known to stigmatize patients who request pain management medications and thus may be difficult to establish future trust. Part of it may be avoiding harm of feeding into a potential addiction to opioids, however, physicians may be causing more harm in the short term by undertreating pain for patients. ${ }^{9}$ Many CNCP/HRNA patients only want to have adequate pain control, however when they demand or firmly request opioids for instance, it may come across to staff as being a difficult and demanding patient whom they are less likely to take seriously. ${ }^{10}$ Currently, there are no protocols for limit setting of the high risk non-adherent patient population in the inpatient setting. While healthcare systems have some interventions currently in place for opioid misuse and abuse, these are largely centered on actions outside the realm of the hospital. Here, the gaze is shifted to looking at inpatient stays.

The Hippocratic Oath describes the physician's duty to act in the patient's best interest which can be conflicting when describing a situation in which the medical teams feel that it is inappropriate to prescribe opioids to a certain patient population (HRNA patients) based upon their addiction diagnosis or non-diagnosis with actions warranting misuse of opioids or other related medications. This then requires physicians to deny certain opioid or related therapies while inpatient which also causes the moral dilemma/distress of the providers. There are no guidelines that direct or lead providers to deal with inpatient situations on how to set boundaries, rules, and guidelines when a patient may need the gold standard therapy--or the therapy teams would offer any other patient without an addiction, but in this situation cannot because it is unsafe to provide such therapy to the patient, and therefore it cannot be offered.

\section{CASE EXAMPLE}

In order to understand the ethical significance associated with the high risk non-adherent patient population, the following case will illustrate the ethical dilemmas many teams face.

\footnotetext{
${ }^{5}$ Ti, Lianping. Leaving the Hospital Against Medical Advice. Pg. e53-e54

${ }^{6}$ Cohen, Mitchell J. A Clinical ethics approach to opioid Treatment. Pg. 522-523

${ }^{7}$ Bonnie RJ, et al. Pain management and the opioid epidemic. Pg. 3-4

${ }^{8}$ Knight, Kelly R. Opioid pharmacovigilance. Pg. 87-95.

${ }^{9}$ Bonne RJ, et al. Pain management and the opioid epidemic. Pg. 4-5.

${ }^{10}$ Olfson, Mark. Service Use Preceding Opioid-Related Fatality. Pg. 538-544.
} 


\section{THE JOURNAL OF HEALTHCARE ETHICS \& ADMINISTRATION}

Vol. 5 | No. 1 (Spring/Summer 2020)

\section{A. Clinical History}

Leslie Smith [a pseudonym] is a middle-aged woman with a history of bipolar disorder, hepatitis C, intravenous drug use, and has had multiple spinal surgeries to repair a T11 fracture. Upon admission, the patient was noted to have a spinal abscess. The patient has had multiple admissions over the past few months and has been requesting higher doses of pain medications. She has been intermittently refusing antibiotics, physical therapy, nursing assessments, thus causing her infection to be high risk.

Medicine teams, psychiatry, chronic pain, infectious disease, and neurosurgery are all in agreement that escalating doses of pain medication is possibly unsafe for the patient and not in line with the patient's best interests. Per chronic pain reports, patient is non-cooperative with trying non-opioid medications. The concern with an increase dose of opioid medication is that the patient is likely to develop opioid induced hypersensitivity. Patient feels stigmatized and feels that provider bias is motivating these conversations about therapy alternatives.

\section{B. Defining the Ethical Dilemma}

The first dilemma starts with patient autonomy versus professional autonomy. The goals of the patient are conflicting with the goals of the provider. The patient is requesting pain medication that she feels is the only solution to her pain. The physicians have objective criteria to illicit their concerns surrounding why the pain medication is not the most effective route of pain control. The second dilemma surrounds beneficence vs. non-maleficence. Providers have the duty to provide care to the patient that provides benefit for the patient while avoiding treatments that cause more harm than benefit. Teams can use risk/benefit analysis to determine which treatments would be of benefit to the patient with the minimal amount of harm. Interestingly, the goals of the patient and the team are the same, however the conflict lies within the route to meet the goal of pain control.

\section{Resolution and Outcome}

General medicine, medical ethics, behavioral health, nursing, and chronic pain teams simultaneously met with the patient to discuss treatment and care plan options. The teams asked Leslie about her feelings and understanding of her pain management plan. She now asks that her cap be $30 \mathrm{mg}$ of Oxycodone instead of $15 \mathrm{mg}$. The current regimen appears to be effective for the patient based on physical and verbal communication of pain scales. Teams explain that if they continue to increase titrations of pain medication as on previous admissions, the patient becomes less responsive and non-communicative. Additionally, teams explained that increased doses of pain medication offer short-term relief and that escalating opioid use is not without short term and long-term risks. Specifically, long term usage of opioids for chronic non-cancer pain is correlated with worsening Gl function and increased pain. This usage has potential to lead to the development of Narcotic Bowel Syndrome and severe chronic abdominal pain. ${ }^{11}$

The patient expressed her frustration surrounding her pain control and feels as though the teams are stigmatizing her based on her history of intravenous drug use. Teams redirected the conversation to short term and long term goals of the patient related to pain management. Leslie desired to go outside however would refuse physical therapy sessions inpatient. Teams discussed with the patient: timing of pain management with physical therapy related to time of day Leslie would prefer to engage in treatment. This way, teams were hearing the patients concerns and promoting patient autonomy by choosing a safe avenue of supportive care.

The teams and the patient are in agreement with physical therapy in the mornings following coffee per patient's request. The nurse will give her pain medication prior to the start of physical therapy.

After the teams' explanations of the risk of more adverse reactions caused by increasing opioid dosages, repeated hospital admissions, worsening pain cycles, ${ }^{12}$ the patient expressed a willingness to try the new pain plan approach despite skepticism about its effectiveness.

\footnotetext{
${ }^{11}$ Burns, Bracken. Narcotic Bowel Syndrome.

${ }^{12}$ Burns, Bracken. Narcotic Bowel Syndrome.
} 


\section{THE JOURNAL OF HEALTHCARE ETHICS \& ADMINISTRATION}

Vol. 5 | No. 1 (Spring/Summer 2020)

Following the patient and team meeting, Leslie was discharged to a skilled nursing facility on a decreased dosage of pain medication after titration within the hospital. She and the teams agreed upon a care plan for readmission purposes and consistency in her care. Leslie's care was also coordinated with her new chronic pain clinic, psychiatrist, and PCP (primary care physician). The patient has not presented to the ED since the previous admission and has sought opioid replacement therapy since admission as documented in outpatient records.

\section{Communication Approaches to This Case}

How do physicians get the patient to buy into the treatment plan? In our experience, it may be more helpful to start by asking questions to gain information from the patient and truly hear his/her concerns surrounding his/her care. These questions can be as easy as: "How is this hospitalization treating you?"; "What has worked for you in the past?"; "What would be helpful now?" From here, it can be helpful to explore the patient's short-term and long-term goals. ${ }^{13}$ Once the teams have a sense of the patient's short and long term goals, they can construct a more personalized approach to meeting these goals. "It sounds like it is really important to you to go outside." Validate the patient's goal. "I think that's a great goal, let's try to create a treatment plan with the options available to reach your goal."

Always be mindful of how the patient is processing said treatment plan and other information. For instance, with the case example, the patient responded better to receiving information in a slower manner. This allowed the patient to digest the medical information being provided which empowered her to take on a more active role in her care. Otherwise, the provider can ask what was missed and reaffirm her commitment to understanding and addressing the patient's concerns.

Ethically speaking, an ethics consultation service would recommend the teams only offer treatment within the standards of care, that are beneficial and effective (beneficence), and most of all safe for the patient (non-maleficence). Then would also recommend allowing the patient to choose which route of safe and effective care she would like to participate in based upon her goals (autonomy).

\section{E. Applied Methods for HRNA patients}

Some patients may nevertheless continue to demand inappropriate treatment. A time-limited trial of potentially inappropriate therapy may be helpful in some situations, with clear criteria for success or failure agreed upon in advance. We would recommend articulating limit-setting in a patient-centered way: if the treatment is harmful, ineffective, unsafe or non-feasible then the treatment will not be offered. The team can then offer treatment options that are safe and effective to the patient, and the patient can then choose (autonomy) which option best fits their goals.

If the patient refuses all treatment options teams are suggesting, strategy could be:

1. Offer all support avenues for the patient (chronic pain, psych, palliative care, addiction medicine, social work, etc.) to illicit goals of care conversations, and support for patient values (involve multi-disciplinary approach).

2. If all teams have been exhausted, and patient still refusing safe/recommended treatment options:

a. Teams do not need to offer unbeneficial, ineffective and unsafe treatment options when patient demands

b. Teams can accept time based trial periods for questionable success of treatment options so long as the treatment meets 3 criteria:

1. Safe

2. No harm

3. Ineffective (the treatment itself may not effect outcome but may be peace of mind to patient and does not cause harm or unsafe safe environment i.e. vitamin C IV/holistic approaches-essential oils)

c. Can always offer a second opinion or seek transfer to another hospital.

Limits set in this manner, may bridge or allow for a more effective course of treatment with high risk non-adherent patients in the inpatient setting. This allows for patients to buy-in, and to build consensus of the goals of both the patient and medical providers.

\footnotetext{
${ }^{13}$ Blackall GF, et al. Breaking the cycle: How to turn conflict into collaboration.
} 


\section{THE JOURNAL OF HEALTHCARE ETHICS \& ADMINISTRATION \\ Vol. 5 | No. 1 (Spring/Summer 2020)}

\section{CONCLUDING REMARKS}

How does medical staff set safe boundaries with patients, build consensus, and provide effective quality care? The high risk nonadherent patient population elicits a challenge for physicians today. Communication when utilized appropriately, is the best way to set safe boundaries, build consensus, and carry out effective, patient-centered care. By establishing and understanding goals of the stakeholders, both the patient and physician teams can effectively mitigate conflict early on. We recommend, a multidisciplinary team-based approach to HRNA patients. Protocols, and policies can be developed out of this program that can establish an assurance of a stigmatization free environment for this patient population. Institutions can further a higher quality of care for all patients by adopting a HRNA limit setting protocol for their inpatient settings.

\section{BIBLIOGRAPHY}

Cohen, Mitchell J. and Jangro, William C. "A Clinical Ethics Approach to Opioid Treatment of Chronic Noncancer Pain." AMA J Ethics 17, no. 6 (2015): 521-529.

Fenton, Joshua J., Peter Franks, Mitchell D. Feldman, Anthony Jerant, Stephen G. Henry,

Debora A. Paterniti, and Richard L. Kravitz. "Impact of patient requests on provider-perceived visit difficulty in primary care." Journal of general internal medicine 30, no. 2 (2015): 214-220.

Jerant, Anthony, Joshua J. Fenton, Richard L. Kravitz, Daniel J. Tancredi, Elizabeth Magnan,

Klea D. Bertakis, and Peter Franks. "Association of clinician denial of patient requests with patient satisfaction." JAMA internal medicine 178, no. 1 (2018): 85-91.

Knight, Kelly R., Margot Kushel, Jamie S. Chang, Kara Zamora, Rachel Ceasar, Emily Hurstak, and Christine Miaskowski. "Opioid pharmacovigilance: A clinical-social history of the changes in opioid prescribing for patients with co-occurring chronic non-cancer pain and substance use." Social science \& medicine 186 (2017): 87-95.

Kong, Erwin L., and Bracken Burns. "Narcotic Bowel Syndrome." In StatPearls [Internet]. StatPearls Publishing, 2019.

Misak, Cheryl J., Douglas B. White, and Robert D. Truog. "Medically inappropriate or futile treatment: deliberation and justification." In The Journal of Medicine and Philosophy: A Forum for Bioethics and Philosophy of Medicine, vol. 41, no. 1, pp. 90-114. Journal of Medicine and Philosophy Inc., 2015.

National Academies of Sciences, Engineering, and Medicine. Pain management and the opioid epidemic: balancing societal and individual benefits and risks of prescription opioid use. National Academies Press, 2017.

Olfson, Mark, Melanie Wall, Shuai Wang, Stephen Crystal, and Carlos Blanco. "Service use preceding opioid-related fatality." American journal of psychiatry 175, no. 6 (2017): 538-544.

Ti, Lianping, and Lianlian Ti. "Leaving the hospital against medical advice among people who use illicit drugs: a systematic review." American journal of public health 105, no. 12 (2015): e53-e59. 